\title{
Gas1 is a modifier for holoprosencephaly and genetically interacts with sonic hedgehog
}

\author{
Maisa Seppala, ${ }^{1}$ Michael J. Depew, ${ }^{1}$ David C. Martinelli, ${ }^{2}$ Chen-Ming Fan, ${ }^{2}$
} Paul T. Sharpe, ${ }^{1}$ and Martyn T. Cobourne1,3

1Department of Craniofacial Development, Dental Institute, King's College London, London, United Kingdom. 2Department of Embryology, Carnegie Institution, Baltimore, Maryland, USA. ${ }^{3}$ Department of Orthodontics, Dental Institute, King's College London, London, United Kingdom.

\begin{abstract}
Holoprosencephaly (HPE) is a clinically heterogeneous developmental anomaly affecting the CNS and face, in which the embryonic forebrain fails to divide into distinct halves. Numerous genetic loci and environmental factors are implicated in HPE, but mutation in the sonic hedgehog $(S h b)$ gene is an established cause in both humans and mice. As growth arrest-specific 1 (Gas1) encodes a membrane glycoprotein previously identified as a Shh antagonist in the somite, we analyzed the craniofacial phenotype of mice harboring a targeted Gas 1 deletion. Gas $1^{-/-}$mice exhibited microform HPE, including midfacial hypoplasia, premaxillary incisor fusion, and cleft palate, in addition to severe ear defects; however, gross integrity of the forebrain remained intact. These defects were associated with partial loss of Shh signaling in cells at a distance from the source of transcription, suggesting that Gas1 can potentiate hedgehog signaling in the early face. Loss of a single Shb allele in a Gas $1^{-/}$background significantly exacerbated the midline craniofacial phenotype, providing genetic evidence that $S h b$ and Gas 1 interact. As human GAS1 maps to chromosome 9q21.3-q22, a region previously associated with nonsyndromic cleft palate and congenital deafness, our results establish GAS1 as a potential locus for several human craniofacial malformations.
\end{abstract}

\section{Introduction}

Holoprosencephaly (HPE) is a clinically heterogeneous developmental field defect of the CNS, in which the embryonic forebrain or prosencephalon fails to divide into distinct halves $(1,2)$. The underlying brain malformation can have a profound affect upon midline development of the face. In the most severe form of HPE, the forebrain remains as a single undivided vesicle and there is cyclopia, with a single midline eye situated below a rudimentary nose or proboscis and midline clefting of the lip and palate. However, the severity varies in both the brain malformation and the craniofacial features, even among members of the same pedigree. In microform HPE, milder craniofacial features such as ocular hypotelorism, premaxillary agenesis, and solitary median maxillary central incisor (SMMCI) can occur in the absence of defects within the CNS (3). The etiology of HPE is complex, with both environmental and genetic factors being implicated (4). Maternal diabetes, alcohol or drug ingestion, and defects in cholesterol metabolism have all been associated with $\operatorname{HPE}(5,6)$, while a number of candidate genes have also been identified in humans, including sonic hedgehog $(\mathrm{SHH})(7,8)$.

Shh signaling is essential for normal development of the early forebrain, as transcripts are expressed in mesendoderm of the prechordal plate $(9,10)$ and are required for formation of ventral midline structures. $\mathrm{Shb}^{-1-}$ mice have otocephaly, with only a single forebrain vesicle and cyclopia (11). Moreover, mutations in human SHH that have been identified suggest a role in both familial and sporadic HPE $(7,8)$ and represent a substantial proportion of cases demonstrating autosomal-dominant inheritance of this condition (12). Interestingly, the spectrum of phenotypic severity character-

Nonstandard abbreviations used: Gas1, growth arrest-specific 1; HPE, holoprosencephaly; Ptc1, Patched1; Shh, sonic hedgehog.

Conflict of interest: The authors have declared that no conflict of interest exists.

Citation for this article: J. Clin. Invest. 117:1575-1584 (2007). doi:10.1172/JCI32032. istic of HPE can be seen in association with identical mutations in SHH, even among members of the same pedigree (8). SHH mutation can also lead to SMMCI in the primary or secondary dentition, occurring as an isolated syndrome $(12,13)$ or as a manifestation of HPE (14).

Shh is a member of the hedgehog family of vertebrate signaling molecules and is essential for normal development of many regions within the embryo (15). The versatility of Shh is reflected in a complex signaling pathway, with unique mechanisms of generation, distribution, reception, and intracellular transduction of the signal (16). Reception at target cells is mediated via direct physical interaction with the Patched1 (Ptc1) transmembrane domain protein (17); in the resting state, Ptc1 inhibits transduction, which is only relieved by the binding of ligand (18). This inhibitory activity is indirect, via Ptc1-mediated membrane localization and phosphorylation of Smoothened (Smo), a G protein-coupled receptor whose activity is essential for signal transduction (19). Because Ptc1 is also a direct transcriptional target of signaling, pathway activity is therefore buffered by the relative availability of bound and unbound receptor at the cell surface $(20,21)$. In vertebrates, signaling is mediated and interpreted principally via Gli transcription factors (Gli1-Gli3) through a large complex of intracellular mediators. Gli2 and Gli3 function as both activators and repressors and are the principal transducers of Shh signaling activity (22-24). Gli1 is a sole activator and specific target of Shh transduction, but plays a secondary role in signaling $(23,25)$. The precise events that mediate reception of Shh on the surface of receiving cells are not fully understood. However, several plasma membrane-associated proteins have been identified that can bind Shh and regulate pathway activity in vertebrates. These include Megalin, a low-density lipoprotein receptor (26), the Ig/fibronectin type III repeat transmembrane domain proteins Cdo and Boc $(27,28)$, and the membrane glycoproteins hedgehog-interacting protein $(29,30)$ and growth arrest-specific 1 (Gas1; ref. 31). 
A
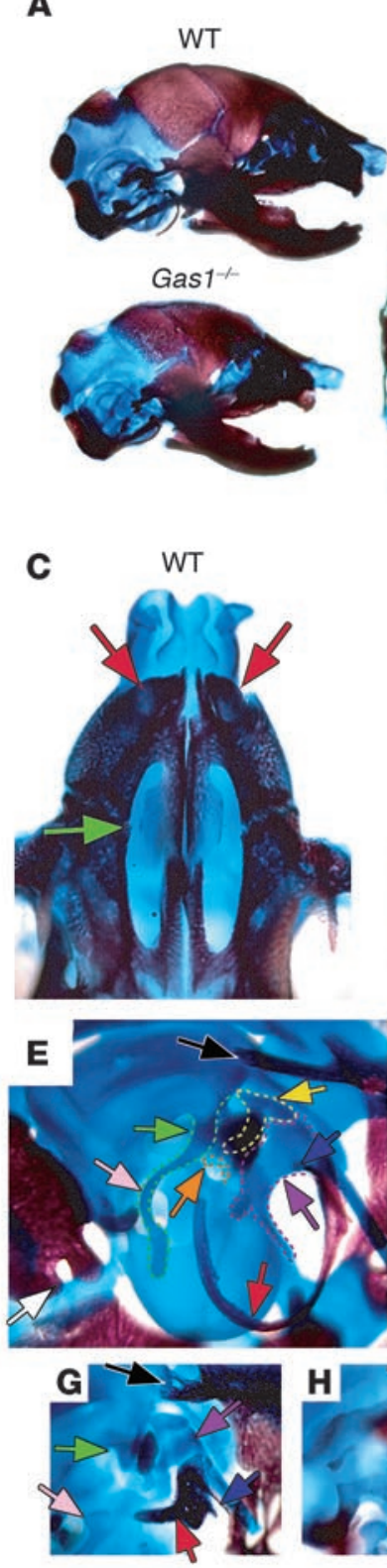

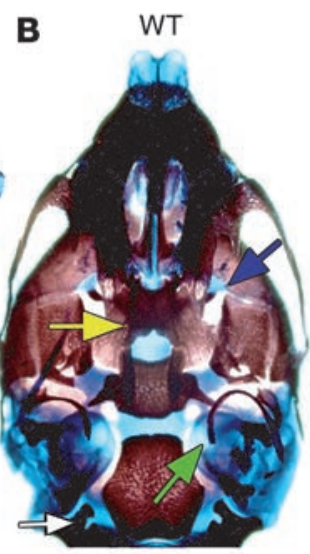

D

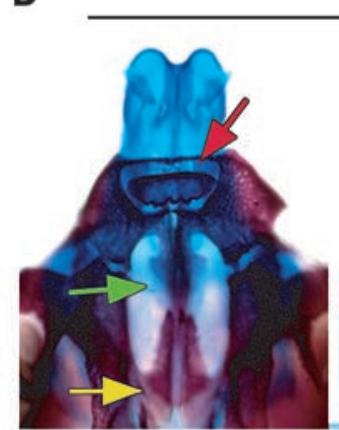

Gas1-
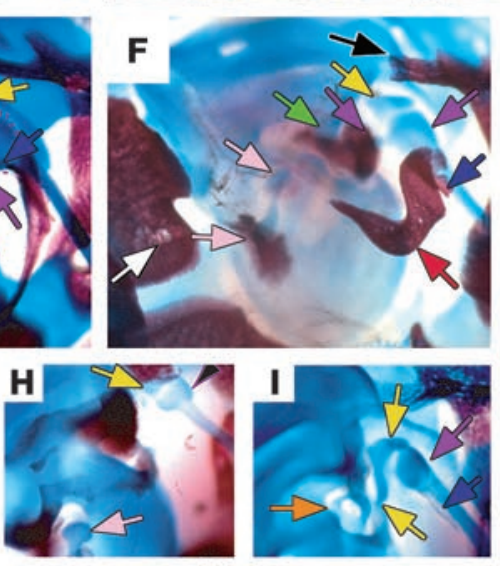

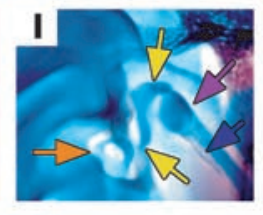

Gas $1^{-1-}$
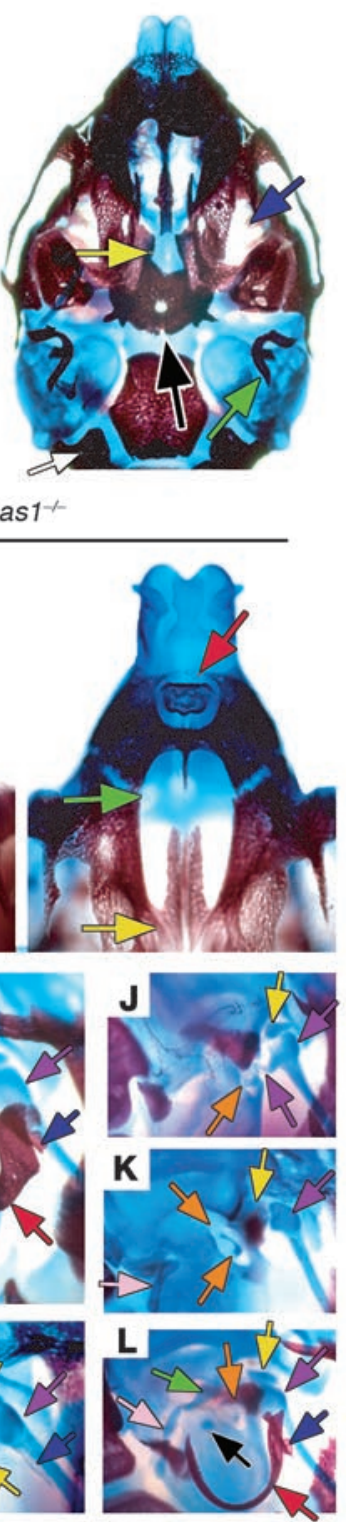

Figure 1

Craniofacial defects associated with the perinatal Gas1-/- skull. (A) Comparison of WT and Gas1-/- skulls differentially stained for bone (red) and cartilage (blue) demonstrated the reduced size of the Gas1-/- skull. (B) Norma basalis of WT and Gas $1^{-1-}$ skulls. Blue arrows indicate the absence of optic pillars in the Gas1-/mouse; yellow arrows highlight the Gas $1^{-/}$cleft palate; black arrow indicates fenestration of the Gas $1^{-/-}$neurocranial base; green arrows indicate dysmorphic ectotympanic rings; and white arrows denote absence of the hypoglossal foramen. (C and $\mathbf{D})$ Magnified norma basalis views of the maxillary and premaxillary palate of WT (C) and $2 \mathrm{Gas1}^{-/-}$(D) neonates without fully cleft palates. Red arrows highlight the morphologic range of fused incisors in $\mathrm{Gas}^{-/-}$mice; green arrows indicate the range of midline hypoplasia in premaxillary palatal shelves and paraseptal cartilages; and yellow arrows show the developing maxillary palatal shelves. $(\mathrm{E}-\mathrm{L})$ Comparison of WT $(\mathrm{E})$ and Gas $1^{-1-}(\mathrm{F}-\mathrm{L})$ middle ear skeletal elements demonstrating the range of dysmorphology evident in Gas 1/- mice. Outlined are the styloid process (green), stapes (orange), incus (yellow), and malleus (purple) in WT mice. Green arrows indicate tympanohyal segments; pink arrows indicate stylohyal segments; purple arrows indicate the mallei; yellow arrows indicate the incuses; blue arrows highlight the gonials; orange arrows highlight the stapes; red arrows indicate the ectotympanics; black arrows indicates ectopic cartilage between the stapes, styloid process, and ectotympanic; and white arrows indicate hypoglossal foreman. Exemplified in $\mathbf{K}$ is the fusion of the stapes to the otic capsule. Original magnification, $\times 1.8(\mathbf{C}$ and $\mathbf{D}) ; \times 2.3(\mathbf{E}$ and $\mathbf{F}) ; \times 1.3(\mathbf{G}-\mathbf{L})$.
Gas1 encodes a glycosylphosphatidyl-inositol-linked membrane glycoprotein that is repressed in response to Shh and has previously been demonstrated in vitro to have an antagonistic effect on Shh signaling in the somites (31). The human homolog of mouse Gas1 maps to chromosome 9q21.3-q22 (32), a locus previously associated with a number of human disorders that involve defects within the craniofacial region, including Chiari type I malformation (OMIM 118420; ref. 33), autosomal-dominant and -recessive neurosensory deafness (OMIM 606705 and OMIM 600974, respectively; refs. 34,35$)$, and nonsyndromic cleft lip with or without cleft palate (36). Moreover, mosaic trisomy 9 has been associated with both HPE (37) and defects of the facial midline in the absence of gross brain malformation (38). Therefore, we analyzed the craniofacial phenotype of mice generated with targeted mutation of Gas 1 and found that they demonstrated features associated with microform HPE. There was lack of development in the maxillary region, fusion of the premaxillary incisors, cleft secondary palate, and pituitary anomalies; however, the telencephalic ventricles remained intact. Surprisingly, these defects were associated with a lack of Shh transduction in cell populations at a distance from the source of transcription, suggesting that Gas1 function can also potentiate signaling in the early face. Indeed, the loss of a single Shb allele in a Gas 1 mutant background significantly worsened the midline craniofacial phenotype, which suggests that Shh and Gas1 genetically interact.

\section{Results}

Gas1 mutants have microform HPE associated with multiple craniofacial defects. Newborn Gas $1^{-/-}$mice exhibit microphthalmia and a lack of retinal pigmentation; despite also being smaller, the gross morphology of $\mathrm{Gas}^{-/-}$heads appears normal (39). In a mixed CD1-129Sv background these mice are viable until adulthood, 


\section{Table 1}

Frequency of midline-centered craniofacial deficiencies in Gas1-1, Gas1--Shh+/, and $\mathrm{Gas}^{1-1-} \mathrm{Shh}^{+-}$mice

\begin{tabular}{lccc} 
Deficiency & $\begin{array}{c}\text { P0 Gas1-/- } \\
(\boldsymbol{n}=\mathbf{1 0})\end{array}$ & $\begin{array}{c}\text { E18.5 Gas1-/-Sh } \boldsymbol{H}^{+/+} \\
(\boldsymbol{n}=\mathbf{5})\end{array}$ & $\begin{array}{c}\text { E18.5 Gas1-/-S } \boldsymbol{S h}^{+/-} \\
(\boldsymbol{n}=\mathbf{4})\end{array}$ \\
Fused premaxillary incisor & $40 \%(4 / 10)$ & $40 \%(2 / 5)$ & $25 \%(1 / 4)$ \\
Synostic premaxilla & $50 \%(5 / 10)$ & $60 \%(3 / 5)$ & $100 \%(4 / 4)$ \\
Complete secondary cleft palate & $30 \%(3 / 10)$ & $20 \%(1 / 5)$ & $100 \%(4 / 4)$ \\
Partial secondary cleft palate & $30 \%(3 / 10)$ & $0 \%(0 / 5)$ & $0 \%(0 / 4)$ \\
Basisphenoid defect & $67 \%(4 / 6)$ & $80 \%(4 / 5)$ & $100 \%(4 / 4)$ \\
Premaxillary incisor agenesis & $0 \%(0 / 10)$ & $0 \%(0 / 5)$ & $75 \%(3 / 4)$ \\
Synostotic mandible/symphysis & $0 \%(0 / 10)$ & $0 \%(0 / 5)$ & $75 \%(3 / 4)$ \\
Fused mandibular incisor & $0 \%(0 / 10)$ & $0 \%(0 / 5)$ & $75 \%(3 / 4)$ \\
Clefting of the basisphenoid & $0 \%(0 / 10)$ & $0 \%(0 / 5)$ & $75 \%(3 / 4)$ \\
Ectopic proximal dentaryA & $0 \%(0 / 10)$ & $0 \%(0 / 5)$ & $100 \%(4 / 4)$ \\
\hline
\end{tabular}

ANot considered a midline-centered deficiency.

while in a mixed 129Sv-C57BL/6 background the majority die within 3 days of birth. At birth, gross morphology of the brain has been reported to be normal in both mutant lines compared with Gas $1^{+/-}$WT mice $(39,40)$.

Because of the putative link between Gas1 function and Shh signaling (31) as well as the pattern of expression (41) and association of the human GAS1 locus with craniofacial anomalies, we analyzed perinatal ontogeny of the craniofacial skeleton in $129 \mathrm{~Sv}-\mathrm{C} 57 \mathrm{BL} / 6$ Gas $1^{-1-}$ mice. Chondrocranial (including both neurocranial and splanchnocranial) and dermatocranial defects were evident, particularly along the neurocranial midline and in the middle and inner ear, but including all 3 primary sensory capsules (nasal, optic, and otic; Figure 1, A and B). These midline defects varied in severity, ranging from bilateral cleft of the secondary palate to absence of elaborated premaxillary palatal processes or failure of the maxillary and palatine palatal processes to fuse in the midline. The premaxillae were hypoplastic, and variable synostosis occurred across the midline, accompanied by the presence of a single premaxillary incisor crown within the premaxillae. While the external nares were patent, the nasal capsular cartilages also exhibited midline defects, such as the absence of elaborated paraseptal cartilages (Figure 1, C and D).

Further defects were identified within the cranial base. The basisphenoid had a large midline basicranial hypophyseal fenestration, usually indicative of abnormal early development at the junction of Rathke's pouch and the anterorostral-most notochord. Laterally, the ala temporali were reduced in size. Overall, varying combinations of midline defects were present in $90 \%$ of mice examined at various stages of development (Table 1). Together, these anomalies all present as features within the clinical spectrum of HPE and in the absence of gross defects within the CNS, suggestive of microform HPE in $\mathrm{Gas}^{-/-}$mice.

These were not the only craniofacial defects. Consistent with the earlier reported ophthalmic deficits (39), the ala hypochiasmatica and associated optic pillars of the optic capsules were lacking in the perinatal $\mathrm{Gas}^{-/-}$skull. The external auditory meatus was hypoplastic or nearly absent in the Gas $1^{-/-}$mouse, and the tympanic rings varied in both size and shape, although they were usually much smaller in diameter and thicker. Gonial bones, when present, were hypoplastic. Defects were also identified in the 3 ear ossicles. In general, the malleus lacked a normal neck, manubrium, and processus brevis and was sometimes synchondrotic with the remnant of the incus. Moreover, a process extended from either the incal or the malleal portion of the fused elements toward the stapes. This process was often synchondrotically fused with the stapes. While the stapes was less affected, it was not completely normal and was occasionally synchondrotic with the otic capsule. The styloid process was always, although variably, affected, typically being markedly hypoplastic and separated into tympanohyal and stylohyal portions. The otic capsule was dysmorphic, as were the middle ear-associated tegmen tympani and retroarticular processes of the squamosals (Figure 1, E-L).

Gas1 and Ptc1 are coexpressed in peripheral regions of hedgehog transduction in the early craniofacial region. Sh signaling is known to be important for normal development of the facial midline $(7,8,42)$. The findings that lack of Gas 1 function is associated with abnormalities in development of the facial midline led us to examine and compare expression domains of Gas 1 and members of the Shh signaling pathway during early development of the facial region.

Shb transcripts demonstrated a very specific temporospatial expression domain in the early craniofacial region. At E10.5 and E11.5, Shb was expressed within the CNS, in the floorplate of the mesencephalon and neuroectoderm of the diencephalon, and in the pharyngeal endoderm and facial ectoderm of the frontonasal process (Figure 2, A, B, F, and G) (42). As targets of Shh signaling, Ptc1 and Gli1 were strongly expressed in these regions, showing a graded distribution of transcriptional activity across fields of responding cells (Figure 2, C, D, H, and I). In contrast, Gas1 was expressed at a distance from the source of $S h b$ transcription, in a domain largely reciprocal to that of Ptc1 and Gli1 (Figure 2, E and J). However, examination of serial sections revealed that regions of coexpression existed among Ptc1, Gli1, and Gas1 along the peripheral margins of their respective expression domains. These overlapping regions of transcription were clearly identifiable at known sites of Shh signaling activity in the craniofacial tissues, particularly the neural tube, first branchial arch, and frontonasal region (Figure 2, C-E and $\mathrm{H}-\mathrm{J}$ ). Given that Shh is able to signal over a distance equal to many cell diameters from the source of transcription $(43,44)$, these findings suggest that Gas1 may interact with Shh in peripheral regions of the signaling domain within the craniofacial region.

Shb signal transduction is reduced in the frontonasal region of $\mathrm{Gas} 1^{-/-}$ mice. We next compared expression of Shh pathway genes in the craniofacial and midfacial regions of WT and Gas $1^{-/-}$mice at E10.5 and E11.5. The domains of Shb transcription were normal at both developmental stages within these regions in Gas $1^{-1-}$ embryos (Figure 2, K, L, O, and P) compared with WT embryos (Figure 2, A, B, F, and G). Ptc1 and Gli1 were expressed in response to this signaling throughout the frontonasal region of WT embryos (Figure 2, C, D, $\mathrm{H}$, and $\mathrm{I}$, arrows); however, in Gas $1^{-/-}$mice there was a clear reduction in the extent of this expression. Ptc1 and Gli1 failed to extend throughout the length of the frontonasal process in $\mathrm{Gas}^{-{ }^{--}}$mice at both stages (Figure 2, M, N, Q, and R, arrows). This reduction was in the peripheral regions of Ptc1 and Gli1 expression, at a distance from the source of $S h b$ transcription and in regions coincident 

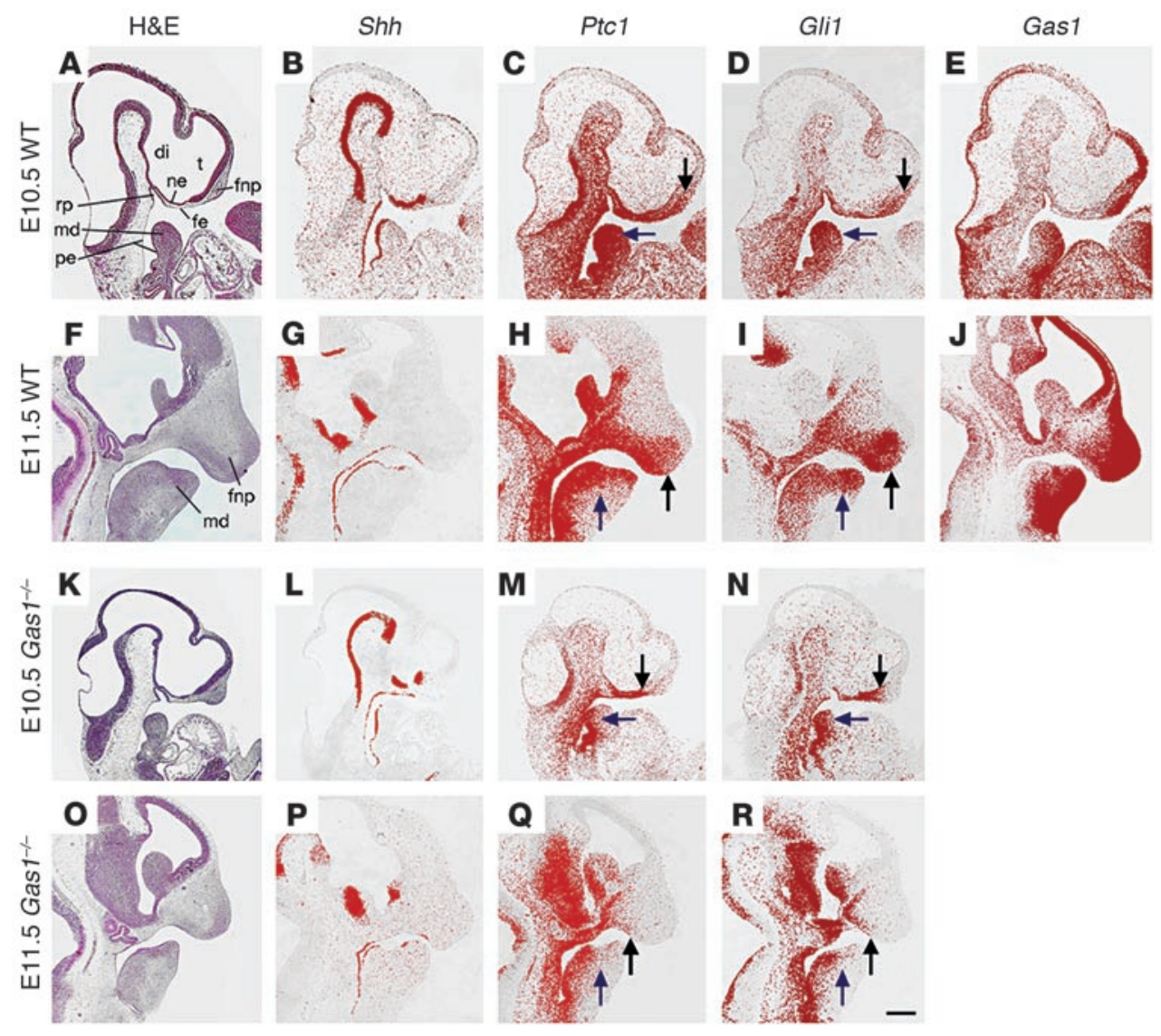

\section{Figure 2}

Shh pathway gene expression in the craniofacial region of WT and Gas1-/- mice. (A, B, F, and G) At E10.5 and E11.5, sagittal sections demarcated Shh in the pharyngeal endoderm, diencephalon, and facial ectoderm of WT embryos. (C, D, H, and I) Ptc1 and Gli1 demonstrated a gradient of transcriptional activity across these regions in response to signaling. ( $\mathrm{E}$ and $\mathbf{J}$ ) In contrast, Gas1 was expressed at a distance from the source of Shh transcription, in a partially overlapping domain with Ptc1 and Gli1, at their peripheral margins. (K-R) In Gas1-1- embryos at both $\mathrm{E} 10.5$ and $\mathrm{E} 11.5$, there was a marked reduction in the domain of both Ptc1 ( $\mathbf{M}$ and $\mathbf{Q}$ ) and Gli1 ( $\mathbf{N}$ and $\mathbf{R}$ ) expression within the frontonasal (black arrows) and mandibular processes (blue arrows) compared with that of WT embryos (compare with respective arrows in $\mathbf{C}, \mathbf{D}, \mathbf{H}$, and I), while Shh expression was normal in Gas $1^{-/-}$ compared with WT embryos (B and G) at both stages. di, diencephalon; fe, facial ectoderm; fnp, frontonasal process; md, mandibular process; ne, neural ectoderm; pe, pharyngeal endoderm; rp, Rathke's pouch; t, telencephalon. Scale bar: $200 \mu \mathrm{m}$. with those expressing Gas1. Gas1 transcripts were only detected at background levels in the Gas $1^{-/-}$mice as a result of the targeting strategy (data not shown and refs. 39, 40). Therefore, a reduction in Shh signaling activity, as assayed by Shh-responsive genes, was identified in the frontonasal region of $\mathrm{Gas}^{-/-}$embryos, suggesting that in regions of coexpression, Gas1 might act as an agonist of Shh signal transduction.

Premaxillary bypoplasia and incisor fusion in Gas $1^{-/-}$mice. At E12.5, a clear restriction in development of the facial midline was identified in $\mathrm{Gas}^{-/-}$embryos. The frontonasal region was narrower, and the early incisor tooth buds were approximated together (Figure 3 , A and B, double arrows). During early tooth development, Shb expression was restricted to these early thickenings of dental epithelium, and in the frontonasal process of WT embryos, these early dental placodes were separated by a region of non-Shb-expressing epithelium (Figure 3, C and E). However, analysis of Shb in the Gas $1^{-/}$mice demonstrated a continuous zone of expression, representing fusion of the incisor placodes (Figure 3, D and F). Furthermore, the response of the frontonasal tissues to Shh signaling was reduced in Gas $1^{-/-}$mice: Ptc1 expression, while normally detectable in the epithelium and mesenchyme of the frontonasal process up to the boundary of the nasal capsule, was not seen in the mutant (Figure 3, G and H). Consistent with these findings, both histological analysis and differential staining of skeletal preparations derived from perinatal Gas $1^{-/-}$mice demonstrated fusion between the premaxillary incisors (see Figure 1D and Figure 3J). Instead of 2 separate tooth germs situated adjacent to the nasal cavity, the $\mathrm{Gas}^{-/-}$mouse had a fused incisor crown positioned below the nasal septum in the midline (Figure 3, I and J). Thus, the single premaxillary incisor crown was a direct result of a lack of development in the facial midline of the Gas $1^{-/-}$embryo.

Reduced Shb signal transduction and cell proliferation in the developing palate of $\mathrm{Gas} 1^{-/-}$mice. A complete or partial cleft of the secondary palate was present in $60 \%$ of $\mathrm{Gas}^{-/-}$mice analyzed (Figure 1 and Figure 4, A and B). Standard histological analysis suggested that the initially vertical palatal shelves elevated above the tongue and oriented themselves into a horizontal position; however, in affected mice these shelves were unable to contact each other in the midline (Figure 4, C-F). By E15.5 in WT mice, the shelves began the process of fusion along the anteroposterior axis, while in $\mathrm{Gas}^{-/-}$mice these shelves failed to meet their counterparts in the midline, resulting in cleft secondary palate.

The Shh signaling pathway is known to play an important role in mediating growth of the palatal shelves $(45,46)$. At E13.5, Shb was strongly expressed in epithelium of WT palatal processes, and Ptc1 exhibited a gradient of activity within the mesenchyme in response to signaling (Figure 5, A, B, E, and F). In addition, Ptc1 was also expressed within bilateral regions of mesenchyme in the bend of the palatal vault (Figure 5F, arrow). We examined Shh signaling activity in palatal shelves of Gas $1^{-/-}$mice at E13.5 and found a noticeable reduction in the range of Ptc1 transcriptional activity within the mesenchyme ( $n=3$ out of 5 mice analyzed); however, $S h b$ was expressed in the palatal epithelium of these mutants (Figure 5, C, D, G, and H). Ptc1 expression was present in close proximity to the palatal shelf epithelium in affected $G a s 1^{-/-}$mice, but the extent of this expression within the mesenchyme was less than that seen in the WT embryos. In addition, Ptc1 expression was absent from palatal bend mesenchyme in Gas $1^{-/-}$mice (Figure 5H, arrow). 

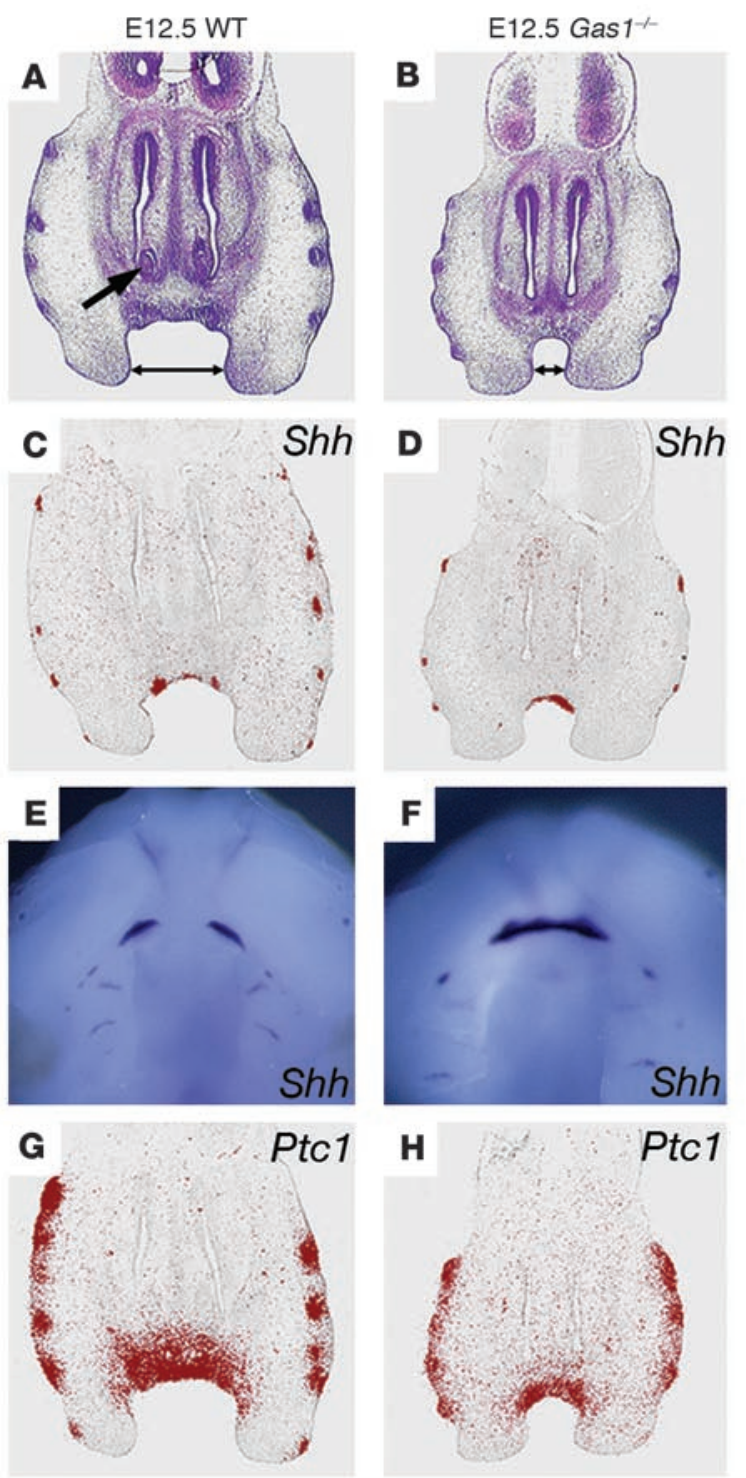

PO WT
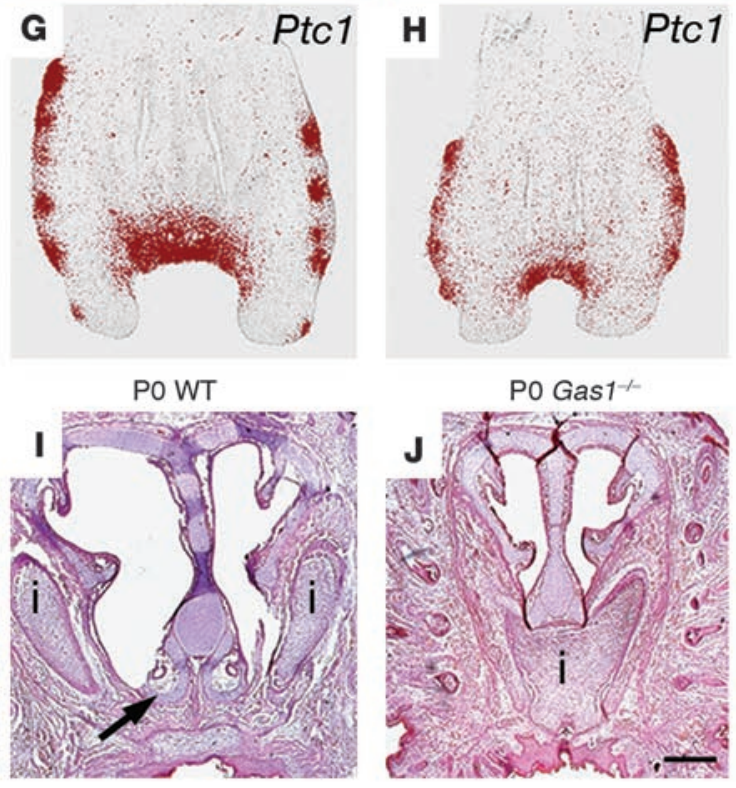

PO Gas $1^{--}$

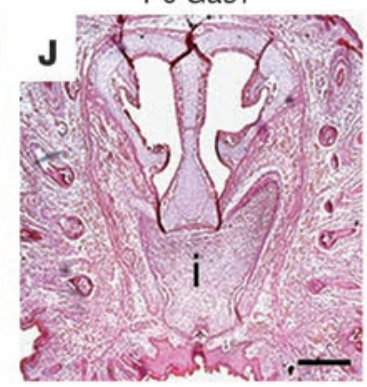

We further addressed the cleft phenotype of Gas $1^{-/-}$mice by analyzing cell proliferation in epithelium and mesenchyme of the palatal shelves at E12.5 and E13.5 (Figure 6, A-F). No statistically significant difference between WT and $\mathrm{Gas}^{-/-}$shelves was detectable at E12.5 (data not shown). At E13.5, the shelves were positioned bilaterally adjacent to the tongue just prior to their elevation above the dorsum. There was no significant difference in epithelial and mesenchymal proliferation within the anterior palate between WT

\section{Figure 3}

The frontonasal region is reduced in Gas1 ${ }^{-/-}$embryos. (A and B) At E12.5, histological analysis revealed a lack of transverse development in the maxillary incisor region in Gas $1^{-/-}$compared with WT embryos (double arrows). In addition, the vomeronasal organ surrounded by the early paraseptal cartilage (A, arrow) failed to develop in Gas $1^{-1-}$ mice. (C-F) This reduced development caused fusion of the early incisor placodes and continuous expression of Shh across the frontonasal region of Gas 1-/- mice. (C and D) Radioactive section in situ hybridization. (E and $\mathbf{F}$ ) Whole-mount in situ hybridization. ( $\mathbf{G}$ and $\mathbf{H}$ ) Ptc1 expression was reduced in the frontonasal region of Gas1-/- compared with WT embryos. (I and J) In perinatal WT embryos, the bilateral maxillary incisor teeth formed in their normal positions adjacent to the nasal capsule, while in Gas $1^{-1-}$ mice, a single enlarged maxillary incisor crown was present, representing fusion between 2 separate tooth germs. The paraseptal cartilage, which failed to form in Gas $1^{-1-}$ mice, is denoted by an arrow. i, premaxillary incisor. Scale bar: $250 \mu \mathrm{m}$ (A-D, $\mathbf{G}$ and $\mathbf{H}) ; 500 \mu \mathrm{m}$ (E and F); $750 \mu \mathrm{m}$ (I and $\mathbf{J})$.

and $\mathrm{Gas} 1^{-/-}$embryos, and while some reduction was seen in epithelium of the apex in the middle and posterior regions, this was not identifiable in the bend region. However, there was a significant reduction in the percentage of mesenchymal cells proliferating within the middle and posterior regions of the palatal shelves at the apex ( $42 \%$ for both; $P<0.001)$ and bend $(40 \%$ and $32 \%$, respectively; $P<0.001$ ) in Gas $1^{-/-}$compared with WT embryos (Figure 6, $\mathrm{G}$ and $\mathrm{H})$. Therefore, in the absence of Gas1 function, a reduction in Ptc1 transcription in mesenchyme of both the palatal apex and bend was associated with a statistically significant reduction in cell proliferation within these regions.

Gas1 and Shb genetically interact. These data suggest that Gas1 is able to positively regulate Shh signal transduction and that this activity is relevant for signaling at a distance from the source of transcription. It is clear that the absence of Gas1 function had important consequences for normal midline development of the craniofacial region. In order to determine whether Gas1 and Shb interact genetically, we analyzed the craniofacial phenotype of mice lacking a single Shb allele in a Gas $1^{-/-}$background.

Individually, both $\mathrm{Gas}^{+/-}$and $S h h^{+/-}$mice were phenotypically normal in the craniofacial region; however, the loss of a Shb allele in a Gas $1^{-/}$background caused a progressively more severe HPE phenotype. Gas $1^{-/-S h h^{+/-}}$embryos survived to birth but only had a single external nostril, while Gas $1^{-/-} S h h^{-/-}$mice had severe craniofacial defects and only survived to around E9.5 (data not shown). Indicative of a significant genetic interaction, $\mathrm{Gas}^{-/-} \mathrm{Shb}^{+/-}$perinatal skulls exhibited exacerbated neurocranial, splanchnocranial, and dermatocranial defects relative to the Gas $1^{-/}$single mutants, as well as significant and novel defects of patterning and development (Figure 7).

Skull size was further reduced in compound $\mathrm{Gas}^{-/-} \mathrm{Shb}^{+/-}$perinatal mice, and patent sutures between the frontal and parietal ossifications were not evident. Development of the neurocranial base was severely disrupted: the trabecular basal plate, which runs from the rostral basisphenoid, presphenoid, and ethmoid through the nasal septum, was discontinuous and cleft. The nasal capsules and associated dermal ossifications were drastically reduced, without proper midline manifestations. The premaxillaries were hypoplastic, synostotic, and lacking teeth. Lateral extensions from the neurocranial base were all disrupted, the ala temporali were hypoplastic, the lamina obturans failed to properly invest the cartilage of the ala, and ectopic preotic cartilaginous pillars extended toward the crista parotica of the otic capsule (Figure 7, A-D). 

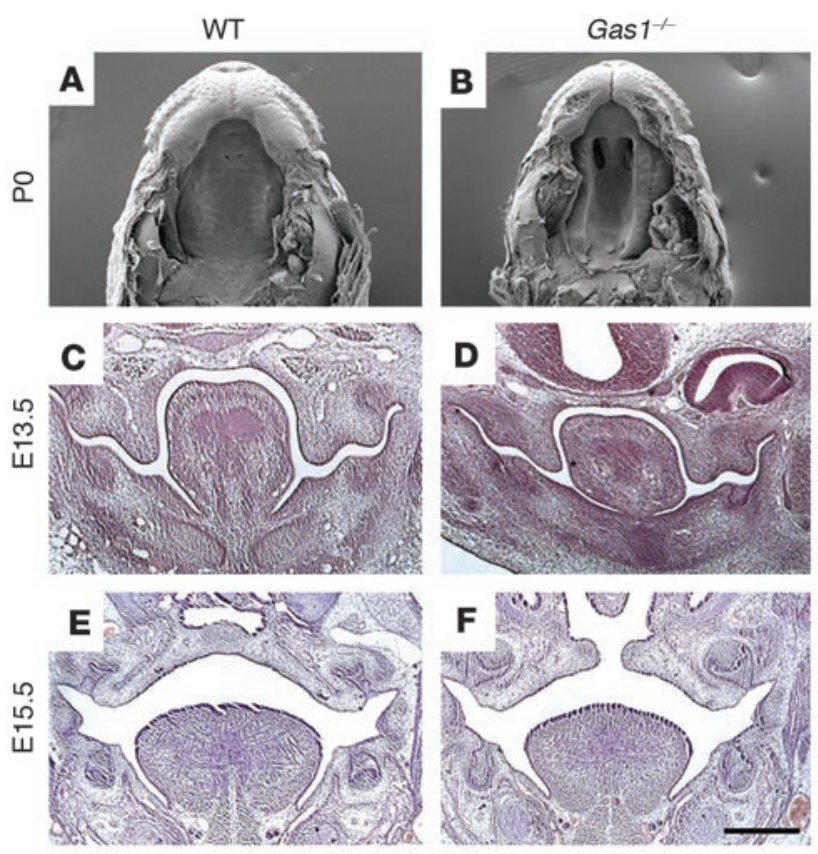

Meckel's cartilage was truncated and lacked a malleal end. Instead, the proximal end bifurcated slightly, apparently being shared by the dysmorphic proximal dentary and an ectopic ossification that appeared to be a mirror-image duplication of the proximal dentary, which included a secondary cartilage-containing condylar process and a molar alveolus. The distal dentary lacked a symphysis, as it was synostotic across the midline, and contained a single lower incisor (Figure 7, C and E). Middle ear-associated splanchnocranial elements either failed to develop (malleus and incus) or were represented by a cartilaginous remnant (stapes). The associated dermatocranial elements, the ectotympanic and gonial bones, also failed to form. The squamosal bones were repatterned and separated into distinct retrotympanic and squamosal portions (Figure 7F). Together, these data demonstrate a marked worsening of the midline craniofacial phenotype with the loss of a single $\mathrm{Shb}$ allele in a Gas1 $1^{-/-}$background.

\section{Discussion}

HPE, cleft palate, and congenital deafness are 3 of the most common craniofacial anomalies that affect human populations. Both cleft palate and congenital deafness have a birth prevalence of around 1 in 1,000 $(47,48)$, while HPE is seen in 1 in 10,000-20,000 live births and in 1 in 250 during embryogenesis, making it the most common cause of brain abnormality in humans $(1,49)$. In many cases the etiology of these conditions remains unknown; however, the 9q21.3-q22 locus has previously been associated

\section{Figure 5}

Shh pathway gene expression in the palatal shelves of WT and Gas $1^{-1-}$ mice. (A-D) At E13.5, Shh was expressed in the epithelium of WT and Gas 1-1- palatal shelves. (E and F) Ptc1 was expressed throughout the epithelium and mesenchyme of WT shelves, in addition to isolated regions in the palatal bend mesenchyme (F, arrow). ( $\mathbf{G}$ and $\mathbf{H})$ In contrast, Gas $1^{-/-}$palatal shelves had reduced Ptc1 expression, which failed to extend along their length and was lost in the palatal bend mesenchyme (H, arrow). Scale bar: $250 \mu \mathrm{m}$.

\section{Figure 4}

Palatogenesis in WT and Gas1-/- mice. (A and B) Scanning electron microscopy demonstrating that palate development was complete in WT mice at PO (A), while there was a full-penetrance cleft of the secondary palate in affected Gas $1^{-1-}$ mice at the same time point (B). (C and D) Histological analysis showed comparable morphology of the palatal shelves at E13.5 in WT and Gas1-l- embryos. (E and F) At E15.5, while the shelves rose above the tongue and fused in the midline in the WT embryos, fusion failed to occur in affected Gas $1^{-/-}$ embryos. Scale bar: $1.25 \mathrm{~mm}$ (A and B); $500 \mu \mathrm{m}(\mathbf{C}-\mathbf{F})$.

with both neurosensory deafness $(34,35)$ and nonsyndromic cleft palate (36), while mosaic trisomy 9 has been implicated in HPE (37) and isolated defects of the facial midline (38). Given that the human GAS1 gene resides at this locus, we examined the consequences of a loss in Gas1 function using a mouse model.

Gas1 was originally identified as a gene highly expressed in a fibroblast cell line maintained under conditions of growth arrest (50); it encodes a membrane glycoprotein that in vitro has been implicated as an inhibitor of cell cycle progression (51,52), a participant in excitotoxic neuronal cell death (53), and a negative regulator of Shh $(31,43)$.However, Gas $1^{-/-}$mice do not demonstrate generalized growth excess or tumor progression: while Gas1 does negatively regulate retinal proliferation (39), it is actually required for normal postnatal proliferation in regions of the cerebellum (40). Similarly, the phenotype of these animals is not indicative of excess Shh signal transduction. The limbs develop with small auto-
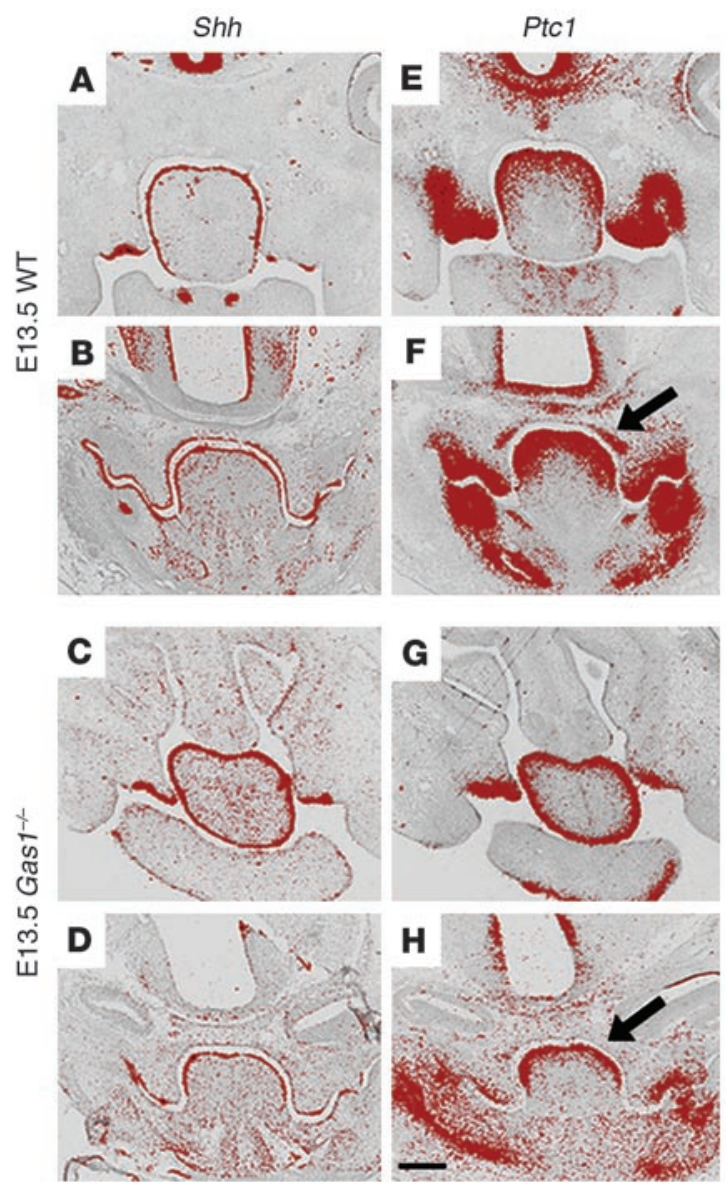

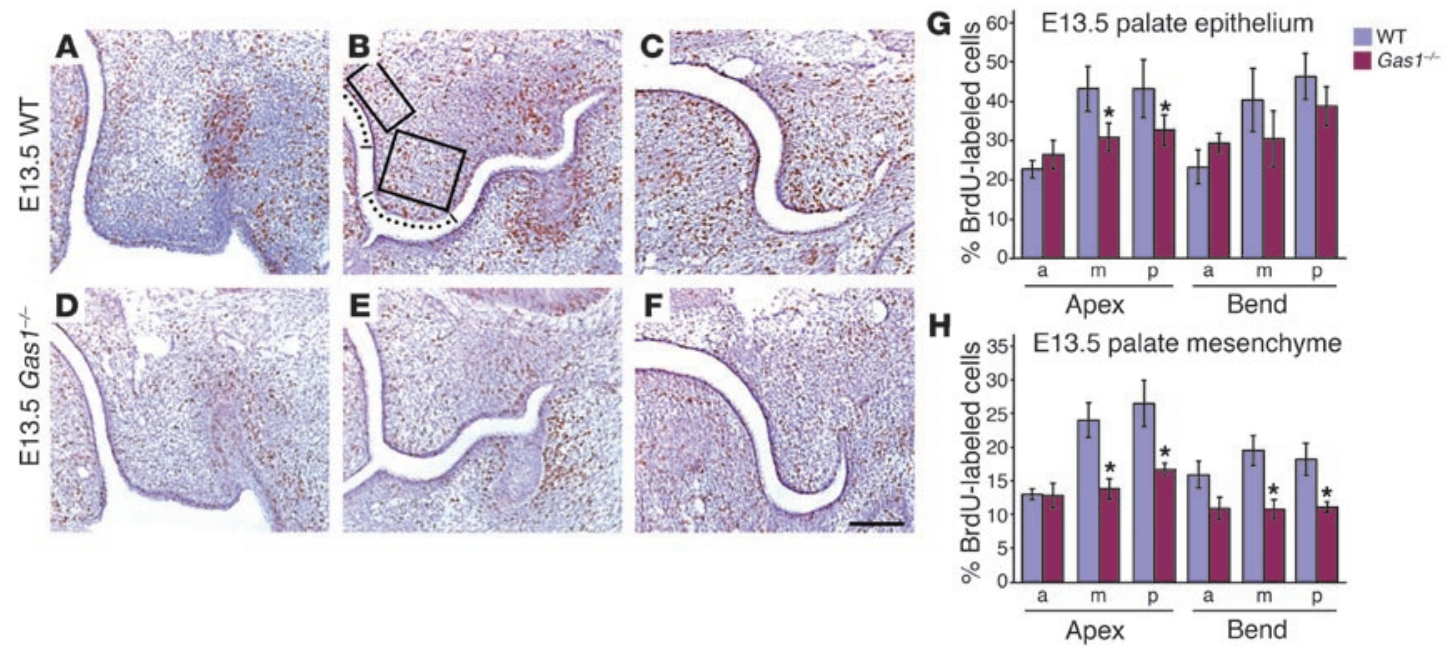

\section{Figure 6}

Cell proliferation in the developing palate of WT and Gas $1^{-/-}$mice. (A-F) BrdU analysis of E13.5 WT and Gas $1^{-/-}$palatal shelves. In B, the upper and lower boxes identify the area of mesenchymal cells counted in the palatal bend and apex, respectively; upper and lower dotted lines represent the regions of epithelial cells counted at the bend and apex, respectively. Scale bar: $125 \mu \mathrm{m}$. (G and $\mathbf{H})$ Percentage of BrdU incorporation for each area assayed. Proliferation rates were significantly reduced in epithelium of the middle and posterior apex and mesenchyme of the middle and posterior apex and bend. a, anterior palate; $\mathrm{m}$, middle palate; $\mathrm{p}$, posterior palate. Data are mean $\pm \mathrm{SD}$. $n=4$ mice per group analyzed for each embryonic stage (total of 45 WT and 55 Gas $1^{-1-}$ sections counted). ${ }^{*} P<0.05$ versus WT.

pods, missing phalanges, and anterior digit syndactyly, but this is secondary to defective proliferation in the apical ectodermal ridge (54). Gas1 also exhibits structural homology to the GFR- $\alpha$ receptor for the GDNF family of ligands (GFL), capable of independently binding the receptor tyrosine kinase ret and interfering with signaling (55). Therefore, Gas1 would appear to have multiple functions, influencing the cell cycle in a cell context-dependent manner and potentially interacting with several signaling pathways.

Our findings suggest that Gas1 can also act cooperatively during Shh signaling in both the early facial processes and developing palate. Gas1 demonstrates coexpression with Ptc1 in peripheral domains of Shh-mediated signal activity; it is within these regions that signaling is reduced in Gas $1^{-/-}$mice. This is consistent with previous reports that the active signaling form of Shh can bind Gas1 (31). This interaction is known to occur through a common interface with that for Ptc1, inviting speculation that Gas1 might act as a coreceptor, presenting Shh to Ptc protein in regions of coexpression (56). However, our present data are inconsistent with previous reports that Gas1 activity can attenuate the mitogenic response to Shh signaling in the somite and downregulate Ptc1 induction in isolated diastema mesenchyme of the jaw $(31,43)$. However, these contrary observations might be explained within the context of endogenous Ptc1 and Gas1 protein levels, which would be expected to strongly influence signal output. From our analysis, it is clear that Gas 1 positively influences hedgehog transduction at the margins of signal activity, where membrane quantities of Gas 1 and Ptc1 are at minimal levels. In regions where either Gas1 or Ptc1 are in gross excess, these effects would appear to be negated.

Gas $1^{-/-}$mice demonstrated a number of features associated with HPE, each with varying levels of penetrance. The evidence of reduced hedgehog target transcriptional activity in the craniofacial region of these mice is consistent with Gas1 mediating its effects through the Shh pathway. HPE is a common congenital anomaly, which in the most severe form is characterized by a com- plete failure of forebrain division and cyclopia (57). However, the phenotypic spectrum associated with this condition is extensive, and a gradation of severity exists in the malformations that can affect both the brain and the face (58). The basis of this clinical heterogeneity is not fully understood, but it has been suggested that HPE represents a multifactorial condition (even in cases demonstrating autosomal-dominant inheritance patterns), as penetrance of the phenotype reflects the varying contributions of multiple genetic and environmental influences within the individual (3). Indeed, the association between hedgehog signaling and HPE provides clear evidence of this complexity. The early craniofacial region is sensitive to perturbations in Shh signaling and this changes with time; disruption of the pathway during discrete periods of embryonic development can recapitulate the phenotypic spectrum of HPE, inducing hypotelorism, midfacial hypoplasia, facial clefting, or frank cyclopia, depending on the timing and magnitude of signal loss (42). In addition, modifying loci have also been identified that act in concert with $S H H$ in the induction of HPE in human populations; these include TGIF and ZIC2 (12). Here, we provide evidence to suggest that GAS1 might represent a further locus for HPE in humans.

We also analyzed the palatal phenotype of Gas1 mutants and provided further evidence that Shh signaling plays an important role in mediating secondary palatogenesis, particularly in mesenchymal proliferation within the palatal shelves (46). Gas $1^{-/-}$mice demonstrated a reduction in the range of Shh signal response across mesenchyme of the palatal shelf. In these mutants, Ptc1 expression remained localized to superficial regions of palatal mesenchyme adjacent to the epithelium and failed to form a normal gradient of activity across the shelves. Given that the cleft phenotype was not completely penetrant, this implies that mesenchymal proliferation must reach a certain threshold to ensure that the shelves elevate above the tongue and meet their counterparts in the midline. The incomplete penetrance associated with the palatal phenotype, in 
A

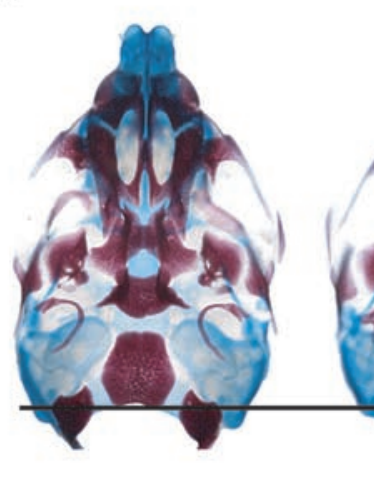

Gas1 $^{-1-S h h^{+/}}$

Gas1 $^{-1} \mathrm{Shh}^{+/}$
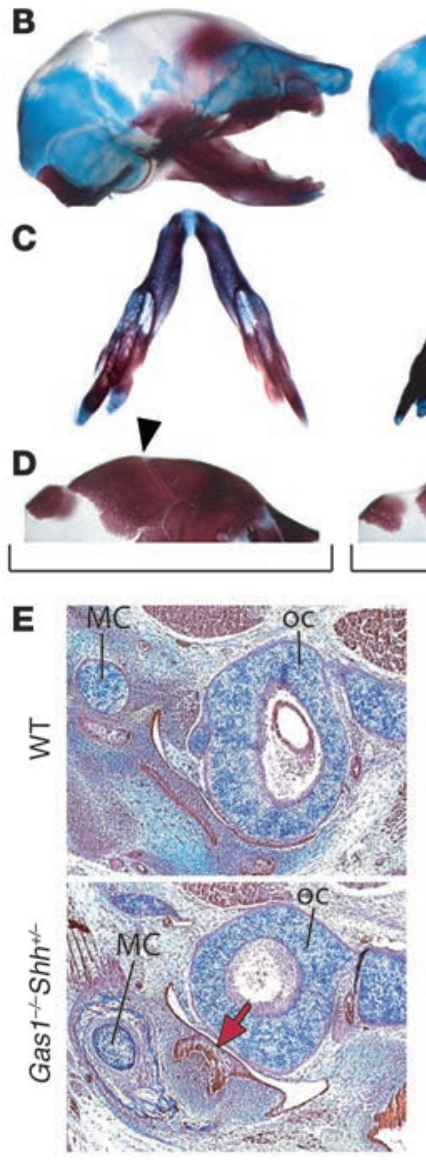
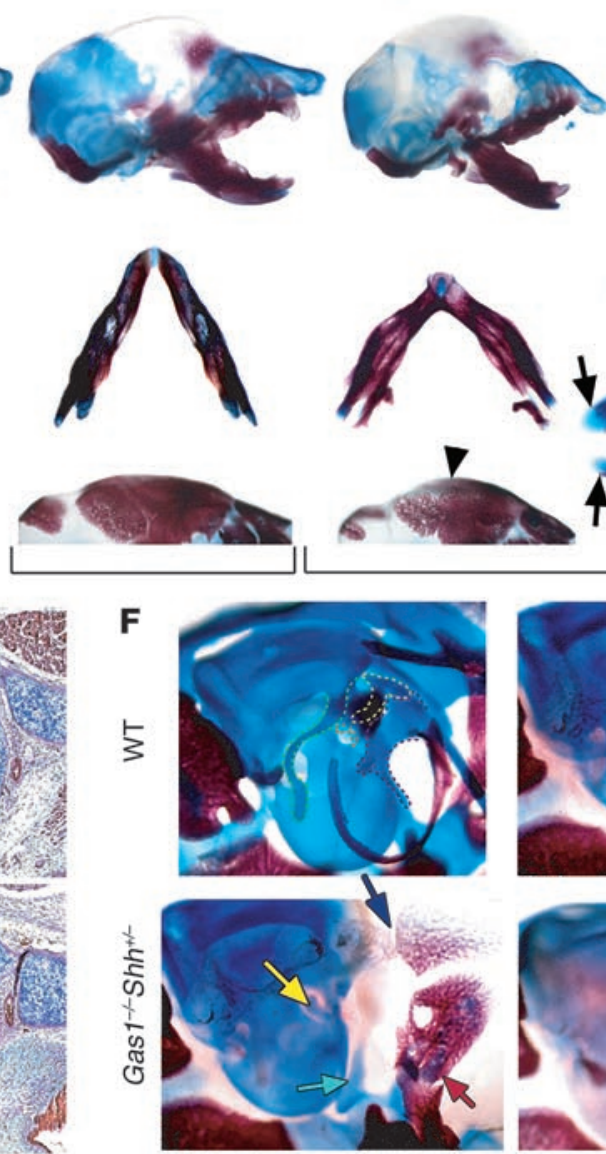
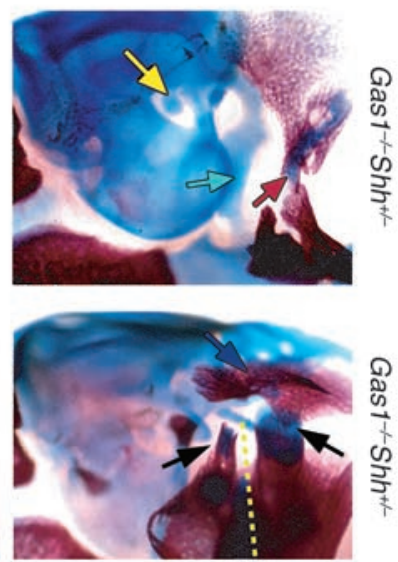

\section{Figure 7}

Demonstration of a genetic interaction between Gas1 and Shh. (A-D) Comparison of Gas1+-Shh+/-, Gas1-/$\mathrm{Shh}^{+/+}$, and $2 \mathrm{Gas1}^{-/-} \mathrm{Shh}^{+/-}$perinatal skulls. The left 3 mice were littermates. (A) Norma basalis. The black line aligns the skulls, which were photographed at the same magnification. (B) Norma lateralis. (C) Dentaries of $\mathrm{Gas1}^{+/-S h h^{+/-}}$, Gas1-/-Shh+/, and

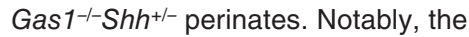
$\mathrm{Gas1}^{-/-} \mathrm{Shh}^{+/-}$perinates, in addition to midline fusion and a single incisor, exhibited what appears to be a duplicated proximal dentary that includes a secondary cartilage-containing condylar process (black arrows) and an alveolus containing an ectopic molar (green arrow). White arrowhead indicates truncation and proximal bifurcation of Meckel's cartilage. Original magnification, $\times 4$. (D) Calvaria of

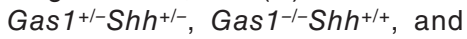
$\mathrm{Gas1}^{-/-} \mathrm{Shh} \mathrm{h}^{+/-}$skulls. Black arrowheads highlight the loss of a patent coronal suture in the calvarium of the Gas1-/-Shh ${ }^{+-}$skull. (E) Histological sections of the developing ectopic molar (red arrow) and dentary. MC, Meckel's cartilage; oc, otic capsule. (F) Comparison of WT and Gas $1^{-/-}$ $\mathrm{Shh}^{+/-}$middle ears. Outlined in the WT image are styloid process (green), stapes (orange), incus (yellow), and malleus (purple). Yellow arrows highlight the vestigial stapes; green arrows indicate the ectopic preotic pillar running from the neurocranial base to the otic capsule; red arrows indicate persistent cartilage of the ala temporalis; blue arrows indicate changes at the squamosal; black arrows indicate the ectopic condyles; dotted yellow line denotes axis of symmetry. Original magnification, $\times 2$. addition to the finding that some $\mathrm{Gas}^{-/-}$mice demonstrating microform HPE were unaffected by cleft palate, indicates that this phenotype can occur independently of HPE and shows the importance of modifying factors in the phenotypic manifestation of this condition. Shh signaling also plays an important role during development of the inner ear: morphogenesis of this region was disrupted in $\mathrm{Shb}^{-/-}$mice, causing a lack of organization into distinct vestibular and cochlear portions and ventral otic derivatives, including the failure of the cochlear duct and cochleovestibular ganglia to develop $(59,60)$. Gas $1^{-/-}$mice exhibited severe disruption in both the middle and inner ear, and it is possible that this is secondary to defective hedgehog signaling.

Importantly, the loss of a single Shb allele in a Gas $1^{-/-}$background significantly worsened the midline craniofacial phenotype and auditory defects of these animals. In particular, the neurocranial base became severely disrupted with a cleft and discontinuous trabecular basal plate, while the premaxillary region lacked incisor teeth. Notably, Gas1 $1^{-/-S h h^{+/-}}$perinates exhibited what appeared to be a duplicated proximal dentary that included a condylar process and an alveolus containing an ectopic molar. This form of patterning defect has not previously been described to our knowledge and suggests an important interaction between Shb and Gas 1 that helps to establish mandibular and hyoid identity during early development of the branchial arches. Our observations, along with the previously documented association between 9q21.3-q22 and congenital malformations of the craniofacial region (34-36), provide strong evidence that the GAS1 locus might act as a modifier for some of these devastating human craniofacial anomalies. 


\section{Methods}

Generation of Gas1 and Shb mutant mice. Gas $1^{-/-}$and $\mathrm{Shb}^{-/-}$mice were generated and genotyped as previously described $(11,39)$ and maintained in a $129 \mathrm{~Sv}-\mathrm{C} 57 \mathrm{BL} / 6$ mixed background. Timed matings were set up such that noon of the day on which vaginal plugs were detected was considered E0.5. All animal procedures were reviewed, approved, and carried out under license issued by the Animals and Scientific Procedures Division of the Home Office, Her Majesty's Government, London, United Kingdom.

Histological and skeletal analysis. For histological analysis, embryos were fixed in $4 \%$ paraformaldehyde at $4^{\circ} \mathrm{C}$, dehydrated through a graded ethanol series, embedded in paraffin wax, sectioned at $7 \mu \mathrm{m}$, and stained with H\&E. For differential staining of bone and cartilage, E18.5 and newborn (i.e., P0) mice were fixed overnight in 95\% ethanol, skinned, and eviscerated. Cartilage staining was carried out by soaking in a solution of $76 \%$ ethanol, $20 \%$ glacial acetic acid, and $0.015 \%$ Alcian blue 8GX (Sigma-Aldrich) for 24 hours, differentiating for 7 days in $95 \%$ ethanol, macerating in $1 \% \mathrm{KOH}$ for 24 hours, and washing overnight under running tap water. Bone staining was carried out by transferring the heads to a freshly made $0.1 \%$ aqueous solution of alizarin red S (Sigma-Aldrich), with the addition of several drops of $1 \% \mathrm{KOH}$ to enhance the darkness of the red color. The samples were then washed for 30 minutes under running tap water, decolorized in $20 \%$ glycerol in $1 \% \mathrm{KOH}$ for 1-2 weeks, and prepared for storage in increasing concentrations of glycerol in $70 \%$ ethanol to a final concentration of $100 \%$ glycerol. Skeletal preparations were photographed in light-field, using a Leica stereomicroscope, while submerged in 100\% glycerol.

In situ bybridization. Whole-mount and radioactive section in situ hybridization was carried out as previously described (61). Whole-mount embryos were photographed on an agarose background suspended in PBS using a Leica stereomicroscope. Light- and dark-field images of sections were photographed using a Zeiss Axioscop microscope and merged in Photoshop CS1 (Adobe).

Proliferation assay. Assays for cell proliferation were carried out using a Zymed BrdU Labeling and Detection Kit (Invitrogen). Mouse embryos were labeled with BrdU via intraperitoneal injection into pregnant females (5 mg/100 g body wt) 2 hours prior to sacrifice. Embryos were fixed in Carnoy's fixative at $4^{\circ} \mathrm{C}$ overnight, dehydrated in methanol, embedded in paraffin wax, and sectioned at $7 \mu \mathrm{m}$. The percentage of BrdU-positive cells was calculated after counting by 1 researcher blinded to experimental group on 2 separate occasions 1 week apart, and the mean value was calculated. A total of 4 WT and 4 Gas $1^{-/-}$mice were analyzed for each embryonic stage, which included a total of $45 \mathrm{WT}$ and 55 Gas $1^{-/-}$sections. BrdU-positive cells were counted in the epithelium and mesenchyme of the anterior and posterior palate using an ocular scale grid orientated at the apex of the palatal projection and in the bend region of the palatal shelf (46). Specifically, at E12.5 this covered an area of mesenchyme that was $0.02 \mathrm{~mm}^{2}$ at the palatal apex and $0.012 \mathrm{~mm}^{2}$ at the bend; these regions were bounded by epithelium of lengths of $132 \mu \mathrm{m}$ and $88 \mu \mathrm{m}$, respectively. At E13.5, the areas of mesenchyme counted were $0.03 \mathrm{~mm}^{2}$ and $0.015 \mathrm{~mm}^{2}$ and bounded by $263-\mu \mathrm{m}$ and $165-\mu \mathrm{m}$ lengths of epithelium at the palatal apex and bend, respectively.

Scanning electron microscopy. For scanning electron microscopy, tissues were fixed and stored in $2.5 \%$ glutaraldehyde in $0.1 \mathrm{M}$ sodium cacodylate buffer, rinsed in $0.1 \mathrm{M}$ cacodylate buffer, and postfixed in $1 \%$ osmium tetroxide in water for 90 minutes, followed by dehydration through a graded series of acetone in water, critical point drying in liquid $\mathrm{CO}_{2}$, and sputter coating with gold. Tissues were examined and recorded in a Phillips SEM501B scanning electron microscope fitted with a Deben Pixie digital scan generator and recorder.

Statistics. Student's $t$ test was used to analyze the significance of the differences in BrdU incorporation rates. A P value less than 0.05 was considered statistically significant.

\section{Acknowledgments}

We thank Andrew McMahon for Shb cDNA, Matthew Scott for Ptc1 cDNA, and Alex Joiner for Gli1 cDNA as well as Tony Brain for scanning electron microscopy. Maisa Seppala is a European Union Marie Curie Early Stage Fellow (grant no. MEST-CT-2004-504025). P.T. Sharpe is supported by the Wellcome Trust. M.J. Depew was supported by the Royal Society.

Received for publication March 6, 2007, and accepted in revised form April 10, 2007.

Address correspondence to: Martyn T. Cobourne, Floor 27, Guy's Hospital, London SE19RT, United Kingdom. Phone: 44-2071884432; Fax: 44-2071884415; E-mail: martyn.cobourne@kcl.ac.uk.
1. Muenke, M., and Beachy, P.A. 2000. Genetics of ventral forebrain development and holoprosencephaly. Curr. Opin. Genet. Dev. 10:262-269.

2. Rubenstein, J.L.R., and Beachy, P.A. 1998. Patterning of the embryonic forebrain. Curr. Opin. Neurobiol. 8:18-26.

3. Ming, J.E., and Muenke, M. 2002. Multiple hits during early embryonic development: digenic diseases and holoprosencephaly. Am. J. Hum. Genet. 71:1017-1032.

4. Ming, J.E., Roessler, E., and Muenke, M. 1998. Human developmental disorders and the Sonic hedgehog pathway. Mol. Med. Today. 4:343-349.

5. Cohen, M.M., Jr. 1989. Perspectives on holoprosencephaly: Part I. Epidemiology, genetics, and syndromology. Teratology. 40:211-235.

6. Cohen, M.M., Jr., and Shiota, K. 2002. Teratogenesis of holoprosencephaly. Am. J. Med. Genet. 109:1-15.

7. Belloni, E., et al. 1996. Identification of Sonic hedgehog as a candidate gene responsible for holoprosencephaly. Nat. Genet. 14:353-356.

8. Roessler, E., et al. 1996. Mutations in the human Sonic Hedgehog gene cause holoprosencephaly. Nat. Genet. 14:357-360.

9. Chang, D.T., et al. 1994. Products, genetic linkage and limb patterning activity of a murine hedgehog gene. Development. 120:3339-3353.

10. Echelard, Y., et al. 1993. Sonic hedgehog, a member of a family of putative signaling molecules, is implicated in the regulation of CNS polarity. Cell. 75:1417-1430

11. Chiang, C., et al. 1996. Cyclopia and defective axial patterning in mice lacking Sonic hedgehog gene function. Nature. 383:407-413.

12. Nanni, L., et al. 1999. The mutational spectrum of the sonic hedgehog gene in holoprosencephaly: $\mathrm{SHH}$ mutations cause a significant proportion of autosomal dominant holoprosencephaly. Hum. Mol. Genet. 8:2479-2488.

13. Garavelli, L., et al. 2004. Solitary median maxillary central incisor syndrome: clinical case with a novel mutation of sonic hedgehog. Am. J. Med. Genet. A. 127:93-95.

14. Muenke, M., et al. 1994. Linkage of a human brain malformation, familial holoprosencephaly, to chromosome 7 and evidence for genetic heterogeneity. Proc. Natl. Acad. Sci. U. S. A. 91:8102-8106.

15. McMahon, A.P., Ingham, P., and Tabin, C. 2003. Developmental roles and clinical significance of hedgehog signalling. Curr. Top. Dev. Biol. 53:1-114.

16. Ingham, P.W., and McMahon, A.P. 2001. Hedgehog signaling in animal development: paradigms and principles. Genes Dev. 15:3059-3087.

17. Goodrich, L.V., Johnson, R.L., Milenkovic, L., McMahon, J.A., and Scott, M.P. 1996. Conservation of the hedgehog/patched signaling pathway from flies to mice: induction of a mouse patched gene by Hedgehog. Genes Dev. 10:301-312.
18. Stone, D.M., et al. 1996. The tumour-suppressor gene patched encodes a candidate receptor for Sonic hedgehog. Nature. 384:129-134.

19. Zhang, X.M., Ramalho-Santos, M., and McMahon, A.P. 2001. Smoothened mutants reveal redundant roles for Shh and Ihh signaling including regulation of L/R asymmetry by the mouse node. Cell. 105:781-792.

20. Casali, A., and Struhl, G. 2004. Reading the Hedgehog morphogen gradient by measuring the ratio of bound to unbound Patched protein. Nature. 431:76-80.

21. Chen, Y., and Struhl, G. 1996. Dual roles for patched in sequestering and transducing Hedgehog. Cell. 87:553-563.

22. Bai, C.B., Stephen, D., and Joyner, A.L. 2004. All mouse ventral spinal cord patterning by hedgehog is Gli dependent and involves an activator function of Gli3. Dev. Cell. 6:103-115.

23. Bai, C.B., Auerbach, W., Lee, J.S., Stephen, D., and Joyner, A.L. 2002. Gli2, but not Gli1, is required for initial Shh signaling and ectopic activation of the Shh pathway. Development. 129:4753-4761.

24. Mo, R., et al. 1997. Specific and redundant functions of Gli2 and Gli3 zinc finger genes in skeletal patterning and development. Development. 124:113-123.

25. Park, H.L., et al. 2000. Mouse Gli1 mutants are viable but have defects in SHH signaling in com- 
bination with a Gli2 mutation. Development. 127:1593-1605.

26. McCarthy, R.A., Barth, J.L., Chintalapudi, M.R., Knaak, C., and Argraves, W.S. 2002. Megalin functions as an endocytic sonic hedgehog receptor. J. Biol. Chem. 277:25660-25667.

27. Tenzen, T., et al. 2006. The cell surface membrane proteins Cdo and Boc are components and targets of the Hedgehog signaling pathway and feedback network in mice. Dev. Cell. 10:647-656.

28. Zhang, W., Kang, J.S., Cole, F., Yi, M.J., and Krauss, R.S. 2006. Cdo functions at multiple points in the Sonic Hedgehog pathway, and Cdo-deficient mice accurately model human holoprosencephaly. Dev Cell. 10:657-665.

29. Chuang, P.T., Kawcak, T., and McMahon, A.P. 2003. Feedback control of mammalian Hedgehog signaling by the Hedgehog-binding protein, Hip1, modulates Fgf signaling during branching morphogenesis of the lung. Genes Dev. 17:342-347.

30. Chuang, P.T., and McMahon, A.P. 1999. Vertebrate Hedgehog signalling modulated by induction of a Hedgehog-binding protein. Nature. 397:617-621.

31. Lee, C.S., Buttitta, L., and Fan, C.M. 2001. Evidence that the WNT-inducible growth arrest-specific gene 1 encodes an antagonist of sonic hedgehog signaling in the somite. Proc. Natl. Acad. Sci. U. S. A. 98: $11347-11352$

32. Evdokiou, A., et al. 1993. Localization of the human growth arrest-specific gene (GAS1) to chromosome bands $9 \mathrm{q} 21.3-\mathrm{q} 22$, a region frequently deleted in myeloid malignancies. Genomics. 18:731-733.

33. Boyles, A.L., et al. 2006. Phenotypic definition of Chiari type I malformation coupled with high-density SNP genome screen shows significant evidence for linkage to regions on chromosomes 9 and 15 . Am. J. Med. Genet. A. 140:2776-2785.

34. Jain, P.K., et al. 1995. A human recessive neurosensory nonsyndromic hearing impairment locus is potential homologue of murine deafness $(\mathrm{dn})$ locus. Hum. Mol. Genet. 4:2391-2394.

35. Kurima, K., et al. 2002. Dominant and recessive deafness caused by mutations of a novel gene, TMC1, required for cochlear hair-cell function.
Nat. Genet. 30:277-284.

36. Marazita, M.L., et al. 2004. Meta-analysis of 13 genome scans reveals multiple cleft lip/palate genes with novel loci on 9q21 and 2q32-35. Am. J. Hum. Genet. 75:161-173.

37. Gerard-Blanluet, M., et al. 2002. Mosaic trisomy 9 and lobar holoprosencephaly. Am. J. Med. Genet. 111:295-300

38. Kaminker, C.P., Dain, L., Lamas, M.A., and Sanchez, J.M. 1985. Mosaic trisomy 9 syndrome with unusual phenotype. Am. J. Med. Genet. 22:237-241.

39. Lee, C.S., May, N.R., and Fan, C.M. 2001. Transdifferentiation of the ventral retinal pigmented epithelium to neural retina in the growth arrest specific gene 1 mutant. Dev. Biol. 236:17-29.

40. Liu, Y., May, N.R., and Fan, C.M. 2001. Growth arrest specific gene 1 is a positive growth regulator for the cerebellum. Dev. Biol. 236:30-45.

41. Lee, C.S., and Fan, C.M. 2001. Embryonic expression patterns of the mouse and chick Gas 1 genes. Mech. Dev. 101:293-297.

42. Cordero, D., et al. 2004. Temporal perturbations in sonic hedgehog signaling elicit the spectrum of holoprosencephaly phenotypes. J. Clin. Invest. 114:485-494. doi:10.1172/JCI200419596.

43. Cobourne, M.T., Miletich, I., and Sharpe, P.T. 2004. Restriction of sonic hedgehog signalling during early tooth development. Development. 131:2875-2885.

44. Gritli-Linde, A., Lewis, P., McMahon, A.P., and Linde, A. 2001. The whereabouts of a morphogen: direct evidence for short- and graded long-range activity of hedgehog signaling peptides. Dev. Biol. 236:364-386

45. Rice, R., Connor, E., and Rice, D.P. 2006. Expression patterns of Hedgehog signalling pathway members during mouse palate development. Gene Expr. Patterns. 6:206-212.

46. Rice, R., et al. 2004. Disruption of Fgf10/Fgfr2bcoordinated epithelial-mesenchymal interactions causes cleft palate. J. Clin. Invest. 113:1692-1700. doi:10.1172/JCI200420384

47. Murray, J.C. 2002. Gene/environment causes of cleft lip and/or palate. Clin. Genet. 61:248-256.
48. Nance, W.E. 2003. The genetics of deafness. Ment. Retard. Dev. Disabil. Res. Rev. 9:109-119.

49. Wilkie, A.O., and Morriss-Kay, G.M. 2001. Genetics of craniofacial development and malformation. Nat. Rev. Genet. 2:458-468.

50. Schneider, C., King, R.M., and Philipson, L. 1988. Genes specifically expressed at growth arrest of mammalian cells. Cell. 54:787-793.

51. Del Sal, G., Ruaro, M.E., Philipson, L., and Schneider, C. 1992. The growth arrest-specific gene, gas 1 , is involved in growth suppression. Cell. 70:595-607.

52. Lee, K.K., et al. 2001. Functions of the growth arrest specific 1 gene in the development of the mouse embryo. Dev. Biol. 234:188-203.

53. Mellstrom, B., et al. 2002. Gas 1 is induced during and participates in excitotoxic neuronal death. Mol. Cell. Neurosci. 19:417-429.

54. Liu, Y., Liu, C., Yamada, Y., and Fan, C.M. 2002. Growth arrest specific gene 1 acts as a region-specific mediator of the Fgf10/Fgf8 regulatory loop in the limb. Development. 129:5289-5300.

55. Cabrera, J.R., et al. 2006. Gas1 is related to the glial cell-derived neurotrophic factor family receptors alpha and regulates Ret signaling. J. Biol. Chem. 281:14330-14339.

56. Mullor, J.L., and Ruiz i Altaba, A. 2002. Growth, hedgehog and the price of GAS. Bioessays. 24:22-26.

57. Golden, J.A. 1998. Holoprosencephaly: a defect in brain patterning. J. Neuropathol. Exp. Neurol. 57:991-999.

58. Ming, J.E., and Muenke, M. 1998. Holoprosencephaly: from Homer to Hedgehog. Clin. Genet. 53:155-163.

59. Liu, W., et al. 2002. Sonic hedgehog regulates otic capsule chondrogenesis and inner ear development in the mouse embryo. Dev. Biol. 248:240-250.

60. Riccomagno, M.M., Martinu, L., Mulheisen, M., Wu, D.K., and Epstein, D.J. 2002. Specification of the mammalian cochlea is dependent on Sonic hedgehog. Genes Dev. 16:2365-2378.

61. Wilkinson, D.G. 1992. In situ bybridisation: a practical approach. IRL Press. Oxford, United Kingdom. 224 pp. 